\title{
FRUTOS DE LA COOPERACIÓN NACIONAL E INTERNACIONAL. EL CASO DEL CONVENIO IDIAPIFCALCIAT EN PANAMA $(1982-1988)^{1}$
}

\author{
César P. Martínez $z^{2}$ Ezequiel Espinosa ${ }^{3}$, Luis E. Berrio ${ }^{4}$, Ariel E. Jaen ${ }^{5}$, Eric Batiste/ ${ }^{6}$, Jaime Gaona ${ }^{5}$, Hernán Gutiérrez $^{6}$
}

\begin{abstract}
RESUMEN
Frutos de la cooperación nacional e internacional. El caso del convenio IDIAPIFCAICIAT en Panamá (19821988). En 1982 se firmó un convenio entre el Centro Internacional de Agricultura Tropical (CIAT), con sede en Palmira, y dos instituciones oficiales panameñas de investigación agrícola: el Instituto de Investigaciones Agropecuarias de Panamá (IDlAP) y la Facultad de Ciencias Agropecuarias (FCA) de la Universidad de Panamá. Este convenio se realizó entre 1982 y 1988 con el objetivo principal de obtener germoplasma mejorado de arroz para las condiciones de riego y secano favorecido. Este trabajo analiza el desarrollo de dicho convenio y los logros alcanzados. Se identificaron sitios claves para las evaluaciones de los. materiales segregantes (FrF8) Y se estableció un flujo del material genético el cual unía los distintos sitios de selección en Panamá con los programas de arroz del ClAT y las pruebas internacionales de arroz de América Latina y el Caribe. Se evaluaron 38305 líneas segregantes, incluyendo viveros internacionales; el porcentaje promedio de selección fue del $13 \%$. La susceptibilidad a las principales enfermedades y a la sequía fueron los factores mas importantes de selección. Once variedades liberadas para diversos ecosistemas en siete países se originaron en este proyecto. Estos resultados indican la importancia de la colaboración interinstitucional e indican que los beneficios obtenidos se extendieron mas allá de las fronteras y tiempo contemplados inicialmente en este proyecto.
\end{abstract}

\begin{abstract}
Fruits of the national and international cooperation. The IDIAPIFCAICIAT agreement in Panamá (19821988). An agreement was signed in 1982 between the International Center for Tropical Agriculture (CIAT) in Palmira, and two official Panamanian institutions for agricultural research: the Panamanian Institute for Agricultural Research (IDIAP), and the University of Panamas Agricultural Sciencies Faculty (FCA). This project was carried out between 1982 and 1988, and its main purpose was to obtain improved rice germplasm under the irrigation and normal conditions prevailing in the zone. The development and achievements of this project are discussed in this documento Key sites for evaluations of segregating genotypes (F2-F8) were identified, and a gene flow was established which joined the different selection sites in Panama with the CIAT rice projects and the international rice experiments in Latin America and the Caribbean. A total of 38.305 segregating lines were evaluated, including international nurseries. The selection average percentage was $13 \%$. Susceptibility to the main diseases and drought stress were the most selective important factors for germplasm failure. These results show the importance of interinstitutional cooperation. The results obtained went beyond the borders and time-frame that were initially established for this project.
\end{abstract}

1 Trabajo presentado en la XLIII Reunión Anual del PCCMCA en Panamá, 1997.

2 Programa de Arroz. CIAT. A.A. 6713. Cali, Valle. Colombia

3 Programa de Arroz IDlAP/FCA. Panamá.

4 INGER-LAC. A. A. 6713. Cali, Valle. Colombia

5 FCA. Estafeta Universitaria. Panamá.

6 IDlAP. Apartado 6-4391, El Dorado. Panamá. 


\section{INTRODUCCIÓN}

$\mathrm{El}$ arroz constituye la base nutricional de gran parte de la población mundial; se estima que el arroz aporta el $20 \%$ de las calorías y el $15 \%$ de la proteína consumida por persona. Se calcula también que en el año 2.025 vivirán alrededor de 8,3 billones de personas en la tierra y que el $50 \%$ de ellas consumirá este cereal.

Según datos estadísticos de la FAO, en el mundo se siembran 151 millones de hectáreas con arroz las cuales producen 567 millones de toneladas métricas. La producción de arroz de América Latina y el Caribe (LAC) representa el 3,6\% (20,5 millones) de ese total y proviene en un $81 \%$ del sector riego; el $93 \%$ de este sector está sembrado con variedades modernas de porte enano. Según Sanint (1996) los avances en tecno-logía para arroz riego en LAC durante las tres últimas décadas han convertido el arroz en un producto básico de la economía y de la alimentación a tal punto que esta región es prácticamente autosuficiente en arroz.

Los avances logrados le han conferido a la producción de arroz una gran estabilidad y confiabilidad en términos de seguridad alimentaria; no obstante, el rápido aumento de la población a nivel mundial y regional está poniendo una gran presión sobre los sistemas de producción de alimentos y la utilización y conservación de los recursos naturales. El Instituto Internacional de Investigaciones del Arroz (IRRI), considera que la producción actual de arroz se debe aumentar en un 70\% para satisfacer su demanda en el año 2025. Este aumento de la producción debe lograrse de una manera sostenible; para ello es preciso que se apliquen nuevos modelos de cooperación internacional, regional e institucional, los cuales deben estar acompañados por nuevas estrategias de mejoramiento varietal y de manejo agronómico del cultivo.

Los granos básicos (arroz, frijol, maíz y sorgo) constituyen los pilares de la seguridad alimentaria en Centro América; el arroz en particular, reviste gran importancia en Costa Rica, Honduras, Nicaragua y Panamá, tanto a nivel de producción como en el consumo per cápita. Sin embargo, problemas de diferente índole afectan esta producción.

Con el fin de reducir el efecto de algunos factores limitantes de la producción de arroz en Panamá, se firmó en 1982 un convenio entre el programa de mejoramiento de arroz del Centro Internacional de Agricultura Tropical (CIAT), con sede en Palmira, Colombia, y dos instituciones oficiales panameñas de investigación agrícola: el Instituto de Investigaciones Agropecuarias de Panamá (IDIAP) y la Facultad de Ciencias Agro- pecuarias (FCA), de la Universidad de Panamá. El objetivo principal era obtener germoplasma mejorado para las condiciones de riego y secano favorecido encontradas principalmente en Panamá. Una de las estrategias seguida fue utilizar diferentes localidades para la evaluación de germoplasma, siendo las principales Alanje para las condiciones de secano y Río Hato para riego. Alanje se escogió por la alta presión de enfermedades tales como Pyricularia (Pyricularia grisea), Helmintosporiosis (Helminthosporium oryzae), escaldado (Rynchosporium oryzae) y manchado de grano, en tanto que Río Hato se utilizó para evaluar principalmente el tipo de planta, susceptibilidad a carbón de la hoja (Entyloma sp), esterilidad, ciclo y potencial de rendimiento.

La importancia de este convenio para Panamá radicaba en la necesidad de mejorar la productividad del arroz a través de mejores variedades y para el CIAT, dicho convenio representó una oportunidad para descentralizar las actividades de mejoramiento varietal y generar germoplasma mejorado no solo para satisfacer las necesidades del programa local, sino también la de los otros programas del área centroamericana y del Caribe. Más aún, buscaba la complementación de las actividades y ventajas comparativas de cada institución participante.

Este convenio colaborativo se financió inicialmente (1982-1986) con fondos provenientes de un préstamo que la Agencia Internacional para el Desarrollo había concedido al IDIAP y posteriormente con fondos del CIAT. Tanto el IDIAP como la FCA aportaron personal científico y auxiliar, apoyo logístico y varias estaciones experimentales.

\section{MATERIALES Y MÉTODOS}

\section{Sitios de evaluación y selección}

Con miras a obtener materiales con amplio espectro de resistencia a enfermedades, insectos, problemas edáficos y climáticos, fue necesario identificar sitios representativos de los principales limitantes de la producción para someter los materiales genéticos a una presión de selección adecuada, constante y uniforme. En el Cuadro 1 se presentan los resultados del análisis físicoquímico de suelos de los campos experimentales utilizados en Panamá; las principales características son:

\section{Alanje:}

Localidad ubicada en el sudeste de la Provincia de Chiriquí con suelos derivados de cenizas volcánicas, con bajo contenido de fósforo (2,9 ppm) y con un poder de fijación de fosfatos estimado en un $80 \%$. La tex- 
Cuadro 1. Resultados del análisis físico-químico de suelos* de los campos experimentales de Alanje, Río Hato, Centro de Estudios e Investigación Agropecuario de Tocumen (CEIAT) y del Centro de Estudios e Investigación Agropecuarias de Chiriquí (CEIACHI).

\begin{tabular}{|c|c|c|c|c|c|}
\hline \multirow[b]{2}{*}{ Característica } & \multirow[b]{2}{*}{ Alanje } & \multirow[b]{2}{*}{ Río Hato } & \multirow[b]{2}{*}{$\begin{array}{c}\text { CEIAT } \\
\text { Tocumen }\end{array}$} & \multicolumn{2}{|c|}{$\begin{array}{c}\text { CEIACHI. David } \\
\text { Clase de suelo }\end{array}$} \\
\hline & & & & $\begin{array}{l}\text { Inseptisol } \\
\text { (aluvión) }\end{array}$ & $\begin{array}{c}\text { UItisol } \\
\text { (residual) }\end{array}$ \\
\hline Color & Pardo oscuro & Pardo claro & Pardo & Pardo-am.-claro & Amarillo rojizo \\
\hline Textura & Franco-arenoso & Franco-arcilloso & Franco-arcilloso & Franco-arcilloso & Franco \\
\hline $\mathrm{pH}$ & 5,5 & 6,3 & 6,1 & 5,1 & 4,0 \\
\hline Materia orgánica (\%) & 12,8 & 2,0 & 4,8 & 4,0 & 4,1 \\
\hline Fósforo (ug/m1) & 2,9 & 11,0 & 7,0 & 3,5 & trazas \\
\hline Potasio (ug/ml) & 88,7 & 157,0 & 210,0 & 120,0 & 112,0 \\
\hline Calcio (meq/100 ml) & 2,6 & 6,2 & 17,5 & 9,9 & 1,7 \\
\hline Magnesio (meq/100 ml) & 1,0 & 1,3 & 7,1 & 5,2 & 1,0 \\
\hline Aluminio (meq/100 m) & trazas & 0,1 & trazas & 0,3 & 3,2 \\
\hline
\end{tabular}

*Laboratorio de suelos IDIAP.

tura de estos suelos es franco-arenosa y son relativamente planos con buen drenaje. El contenido de potasio y de materia orgánica es de mediano a alto, con una reacción ligeramente ácida. La precipitación es mayor de $2.000 \mathrm{~mm}$ al año y bien distribuida. La temperatura media anual es de $27^{\circ} \mathrm{C}$ con una humedad relativa media del $83 \%$. Estas condiciones favorecen la presencia de las principales enfermedades del arroz.

\section{CEIACHI:}

Centro de Estudios e Investigaciones Agropecuarias de Chiriquí, ubicado en las cercanías de David, Provincia de Chiriquí. Allí se distinguen dos tipos de suelos: Inseptisol y Ultisol. El suelo Inseptisol es de origen aluvial, de textura franco-arcillosa, con buena retención de humedad, reacción ligeramente ácida y relativamente fértil, a excepción del bajo contenido de fósforo. El suelo Ultisol es de origen residual, de textura franca, buen drenaje, reacción extremadamente ácida $(\mathrm{pH}=4, \mathrm{O})$, con un alto contenido de aluminio y muy deficiente en fósforo. Al igual que Alanje la precipitación es superior a los $2.000 \mathrm{~mm}$ anuales, siendo octubre el mes mas lluvioso. La temperatura media anual es 27,4 ${ }^{\circ} \mathrm{C}$ y una humedad relativa media del $79 \%$. Estas condiciones favorecen el desarrollo de las principales enfermedades foliares del arroz.

\section{CEIAT:}

Centro de Estudios e Investigaciones Agropecuarias de Tocumen, ubicado cerca del Aeropuerto Internacional de Tocumen, Provincia de Panamá. El suelo es de textura franco-arcillosa, con drenaje imperfecto, li- geramente ácido y de mediana fertilidad. La precipitación es de $1.500 \mathrm{~mm}$ entre mayo y diciembre, siendo octubre el mes más lluvioso. La temperatura media es de $26,7^{\circ} \mathrm{C}$ y una humedad relativa media del $83 \%$. En esta localidad la incidencia de Pyricularia es menor en comparación con las otras, pero se presenta buena incidencia del añublo de la vaina (Thanatephorus cucumeris), escaldado (Rhynchosporium oryzae) y carbón de la hoja (Entyloma sp.).

\section{Río Hato:}

Localidad ubicada en la zona costera de la Provincia de Coclé, con suelos de textura franco-arcillosa, ligeramente ácidos y de mediana fertilidad. La precipitación y la humedad relativa son mas bajas que en los otros sitios, condiciones que hacen que la incidencia de enfermedades sea relativamente baja. Inicialmente se utilizó para evaluaciones y selección bajo condiciones de riego y secano; la ocurrencia de períodos prolongados y erráticos de sequía determinó que las evaluaciones en secano fueran suspendidas y trasladadas a David, Chiriquí.

En el Cuadro 2 se clasifican los principales factores limitantes de la producción de arroz en los sitios seleccionados para realizar la evaluación y selección de los materiales genéticos. Como se puede observar, la presión de selección no es igual en todos los sitios; la presión de enfermedades es mucho mayor en Alanje seguida por David. En Río Hato la precipitación total es baja y muy errática, por lo cual se pueden realizar trabajos de selección buscando tolerancia a la sequía. En 
Cuadro 2. Clasificación de los factores limitantes de la producción de arroz bajo condiciones de secano en los sitios usados como centros de evaluación y selección. Convenio IDIAP-FCA-CIAT.

\begin{tabular}{|c|c|c|c|c|c|}
\hline $\begin{array}{l}\text { David } \\
\text { Enfermedades }\end{array}$ & Alanje & a. & b. & Río Hato & Tocumen \\
\hline Pyricularia grisea & $* * *$ & $* * *$ & $* *$ & $* *$ & $* *$ \\
\hline Rynchosporium oryzae & $* *$ & $* * *$ & $* *$ & $*$ & $* * *$ \\
\hline Helminthosporium oryzae & $* * *$ & $* *$ & $* *$ & $*$ & $*$ \\
\hline Manchado de grano & $* * *$ & $* *$ & $* *$ & $*$ & $*$ \\
\hline Acrocylindrium sp & $* *$ & $* *$ & $*$ & $*$ & $* *$ \\
\hline Entyloma sp & $*$ & $*$ & $*$ & $* *$ & $* * *$ \\
\hline Mancha ojival & $* * *$ & $*$ & - & - & - \\
\hline \multicolumn{6}{|l|}{ Suelos } \\
\hline Fertilidad & media & alta & baja & baja & media-alta \\
\hline Aluminio & \multirow[t]{2}{*}{-} & - & $* * *$ & - & - \\
\hline OTROS & & & & & \\
\hline Sequía & $*$ & $*$ & $* *$ & $* * *$ & $*$ \\
\hline $\begin{array}{l}\text { - = Sin ninguna importancia } \\
\text { a. = Lote experimental - vertisol } \\
\text { b. = Sabana - suelos ácidos }\end{array}$ & \multicolumn{3}{|c|}{$\begin{array}{l}*=\text { Severidad baja } \\
* *=\text { Severidad media } \\
* * *=\text { Severidad alta }\end{array}$} & & \\
\hline
\end{tabular}

Tocumen se presentan ocasionalmente otras enfermedades como el añublo bacterial (Xanthomonas sp.)

\section{Flujo del material genético}

La Figura 1 presenta el flujo del material genético dentro de Panamá y su conexión con el Programa de Arroz del CIAT en Colombia y el IRTP (Programa de Pruebas Internacionales de Arroz para América Latina y el Caribe; ahora llamado INGER-LAC).

Para las condiciones de secano favorecido el proceso empezaba en Alanje con las poblaciones $\mathrm{F}_{2}$ provenientes del CIAT; la $\mathrm{F}_{3}$ se evaluaba en David y la $\mathrm{F}_{4}$ se multiplicaba en Río Hato bajo condiciones de riego; la $\mathrm{F}_{5}$ volvía de nuevo a David y la semilla $\mathrm{F}_{6}$ se dividía en tres partes: una se sembraba en Alanje para reconfirmación de la tolerancia a enfermedades; con las dos partes restantes se sembraban pruebas de observación en Tocumen bajo condiciones de secano y Río Hato bajo riego. Parte de la semilla $\mathrm{F}_{5}$ masal se enviaba a CIAT para evaluaciones en marzo en Santa Rosa y posterior entrega a la red del IRTP, para inclusión en sus viveros internádonales. Para las condiciones de riego las evaluaciones empezaron con la $\mathrm{F}_{2}$ en Río Hato; la $\mathrm{F}_{3}$ se avanzaba en Río Hato y la $\mathrm{F}_{4}$ iba a David para ser evaluada por tolerancia a enfermedades. Las mejores líneas seleccionadas en parcelas de observación en David, Tocumen y Río Hato formaban parte de los ensayos de rendimiento (E.R) y posteriormente de las pruebas regionales (P.R) sembradas en fincas de agricultores localizadas en las zonas productoras más importantes de Panamá.

Dado que los materiales genéticos se evaluaban y seleccionaban bajo buena presión de enfermedades en Alanje y David, se eliminaron las evaluaciones de las poblaciones segregantes tempranas en Tocumen, Chichebre y Río Hato.

Todo material genético distinto a la $\mathrm{F}_{2}$ que entraba por primera vez al proyecto se evaluaba primero en Alanje y luego en David.

\section{Material genético evaluado y seleccionado}

Los materiales genéticos se sometieron a una presión alta y uniforme de enfermedades de acuerdo a los diseños de campo y metodología recomendadas por la sección de Fitopatología del programa de arroz del CIAT; el diseño básicamente consistía en:

a) Siembra de surcos esparcidores de inóculo compuestos por una mezcla de cuatro a seis variedades en igualo diferente proporción. Esta mezcla incluía variedades susceptibles universales, variedades comerciales cuya resistencia ya se había roto y principales variedades sembradas en el área. 


\section{CHIRIQUI}

CEIACHI - DAVID

IDIAP - ALANJE
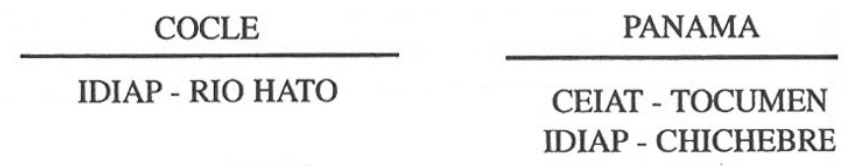

Secano
(may-sep)

Secano

(may-sep)

IDIAP - RIO HATO

IDIAP - CHICHEBRE

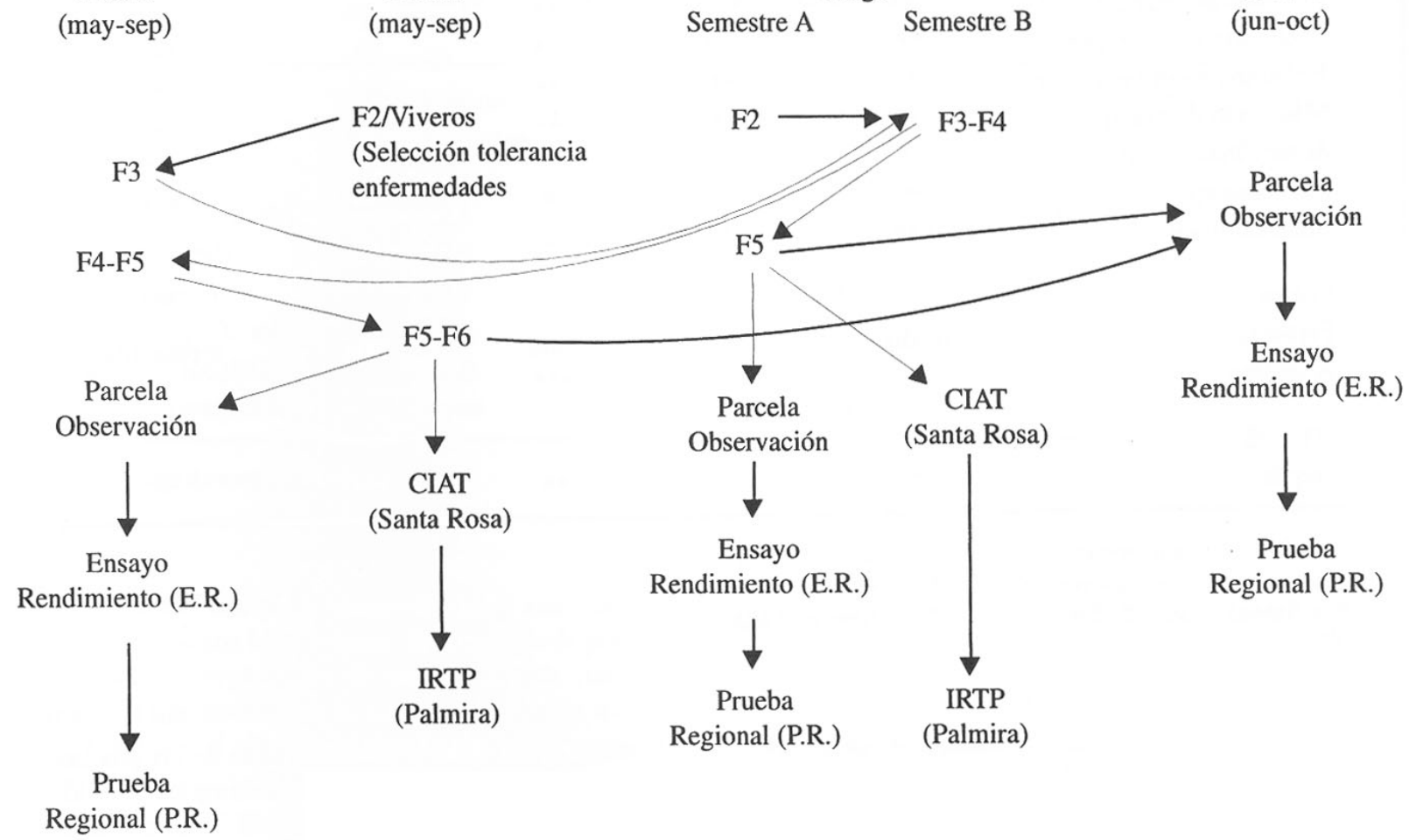

Figura 1. Flujo del material genético en el proyecto colaborativo IDIAP/FCNCIAT.

Los surcos esparcidores se sembraban al menos dos a tres semanas antes que el material genético y en forma perpendicular al sentido de la siembra. Los surcos esparcidores quedaban expuestos al inóculo del ambiente y dada su composición favorecía la multiplicación de los patógenos virulentos existentes.

b) Para promover una distribución uniforme del inóculo en el campo experimental, los surcos esparcidores se inoculaban con hojas infectadas y recibían mayor cantidad de nitrógeno.

c) Variedades susceptibles y resistentes eran sembradas como testigos conjuntamente con el material genético a evaluar cada 20 surcos.

En el Cuadro 3 se resume la cantidad de materiales evaluados y seleccionados durante el período 19821988 en los diferentes sitios utilizados en Panamá.

\section{d) Talleres de mejoradores}

Como se mencionó anteriormente, la importancia de este convenio colaborativo radicaba también en la identificación de germoplasma útil para otros países. Con el fin de promover el intercambio de germoplasma y de información entre los institutos de investigación de la región se realizaron dos talleres de mejoradores (1983 y 1985) en los cuales participaron los líderes de los programas de arroz de los países centroamericanos e investigadores de los cursos de capacitación de arroz del CIAT. Tales talleres fueron patrocinados por el Programa de Pruebas Internacionales de Arroz para América Latina (IRTP-ahora INGER). Para estas actividades se aprovecharon las parcelas experimentales de materiales segregantes establecidas en los dife-rentes sitios, y en donde los participantes tuvieron la oportunidad de evaluar y seleccionar los mate-riales más relevantes para sus países. 
Cuadro 3. Materiales evaluados y seleccionados por el convenio colaborativo IDIAP/ FCA/CIAT en varios sitios durante el período 1982-1988.

\begin{tabular}{|c|c|c|c|c|}
\hline \multirow[b]{2}{*}{ Clase de Material } & \multirow[b]{2}{*}{ Localización } & \multicolumn{2}{|c|}{ Número de líneas } & \multirow[b]{2}{*}{$\%$ de Selección } \\
\hline & & Evaluadas & Seleccionadas & \\
\hline Generaciones & Alanje & 9.175 & 1.265 & 14 \\
\hline \multirow[t]{5}{*}{ Tempranas $\left(\mathrm{F}_{2}-\mathrm{F}_{5}\right)$} & David & 6.909 & 119 & 2 \\
\hline & Río Hato (Sec.) & 9.197 & 526 & 6 \\
\hline & Río Hato (Riego) & 3.204 & 500 & 16 \\
\hline & Tocumen & 1.918 & 1.178 & 61 \\
\hline & Chichebre & 1.589 & 554 & 35 \\
\hline \multirow[t]{5}{*}{ Parcelas Observ. } & Alanje & 1.127 & 44 & 4 \\
\hline & David & 883 & 62 & 7 \\
\hline & Río Hato (Riego) & 1.119 & 267 & 24 \\
\hline & Río Hato (Sec.) & 567 & 75 & 13 \\
\hline & Tocumen & 788 & 106 & 14 \\
\hline \multirow[t]{2}{*}{ Ensayos de Rendimiento } & Riego (Río Hato) & 369 & 124 & 34 \\
\hline & $\begin{array}{l}\text { Secano (Tocumen, } \\
\text { David y Chichebre) }\end{array}$ & 375 & 98 & 26 \\
\hline Viveros Internac. & $\begin{array}{l}\text { Secano (Tocumen, } \\
\text { Alanje, David) }\end{array}$ & 1.042 & 149 & 14 \\
\hline Pruebas Regionales & (Riego y Secano) & 43 & 19 & 44 \\
\hline TOTAL & & 38305 & 5086 & 13 \\
\hline
\end{tabular}

\section{RESULTADOS Y DISCUSIÓN}

En los primeros años el mayor esfuerzo se concentró en la introducción y evaluación de materiales segregantes en diferentes generaciones y en la evaluación de los bancos de germoplasma de arroz de riego y secano favorecido provenientes del CIAT con el fin de identificar progenitores potenciales para un programa regional de cruzamientos.

En el Cuadro 3 se observa que más de 38.000 líneas fueron evaluadas durante los siete años que duróeste convenio. El porcentaje de selección varió del $2 \%$ en David al $61 \%$ en Tocumen, para un promedio gene-ral del $13 \%$. La alta presión de enfermedades en Alan-je y David, así como la baja y errática distribución de las lluvias en Río Hato, fueron los factores mas importantes que influyeron en el descarte de los materiales. Adicionalmente se evaluaron en Alanje 1.042 cultivares del banco de germoplasma del CIAT, se obtuvo un $3 \%$ de cultivares tolerantes a enfermedades y de buenas características agronómicas. Estos resultados y los problemas de mala adaptación a las condiciones locales prevalecientes observados en las generaciones segregantes sugieren el establecimiento de un programa de cruzamientos y selección específicos para la región.

Con la ayuda de la base de datos del Programa de Arroz del CIAT (conformada con la información suministrada por todos los investigadores que participan en INGER-LAC) se hizo un seguimiento sobre la utilización de los materiales seleccionados durante este convenio, los cuales se presentan a continuación:

En 1985 se introdujeron de Río Hato a CIAT-Santa Rosa varias líneas $\mathrm{F}_{4}$ para evaluación a enfermedades fungosas. Allí se hizo selección y se obtuvo semilla $\mathrm{F}_{5}$ la cual fue entregada al IRTP. Esta red completó la caracterización y multiplicación de este germoplasma y al final se incluyeron cuatro de estas líneas en el VIOAL 1986. Una de ellas, la P 3831-F3-RH38-8-1M, proveniente del cruce Oryzica 1/P 1897-15-1-4-1- 
1B/Costa Rica, fue liberada como variedad en 1990 en República Dominicana con el nombre de Juma 64, y en 1991 como Altamira 9 en Nicaragua.

Este mismo VIOAL 1986 formó parte de los viveros internacionales evaluados por el convenio colaborativo IDIAPIFCNCIAT y de allí el programa nacional de arroz de Panamá seleccionó dos líneas, las cuales pasaron a pruebas de observación y ensayo de rendimiento en 1987 y a pruebas regionales en 1988. Estas líneas, la P 3621-F2-1-2-8 del cruce Metica 1/Suakoko/Ceysvoni y P 4721-F2-1O-6 (P 2231-F4-138-6-1B/IR553313-1-1/Metica 1), fueron liberadas por la Facultad de Ciencias Agropecuarias (FCA) de la Universidad de Panamá con los nombres de Panamá 3621 y Panamá 4721, en el año de 1993.

Como resultado del Taller de Mejoradores realizado en 1985, el programa nacional de El Salvador identificó dentro de los materiales seleccionados por su mejorador en dicho taller una línea promisoria, la $\mathrm{P}$ 3299-F4-78-3-1B-1 (P 127 (\};;8M-I-3M-1/IRI909-1-33/IRAT 8), la que fue nombrada como Centa A 5 en el año de 1989.

En el segundo semestre de 1986 se enviaron al CIAT-Palmira 144 líneas avanzadas provenientes de Alanje y Río Hato los que se entregaron al IRTP para su respectiva caracterización, multiplicación y selección; se incluyeron 24 líneas de ellas en el VIOAL 1987B y VIOAL 1988A. Dos de estas líneas ya habían sido identificadas como promisorias en Panamá, las cuales después de ensayos de validación fueron liberadas en $\mathrm{Pa}$ namá en el año de 1987; la P 2062-F4-17-33-1-RHl del cruce CICA 7/S 12-30/P 901-22-11-5-3-2-1B como Panamá 1537, y la línea P 1381-1-8M-2-1B-CH4 (P93122/P 918-25/P 914-43/P 918-25) como Panamá 1048. Otra línea de este grupo la P 4070-F3-3-RH3-7-1BA (P 1386-6-8M-I-3M-1/CamponilTapuripa) fue liberada como Fonaiap 1 en Venezuela en 1993.

Un total de 1.723 líneas (generaciones F3 a F8) seleccionadas durante el año de 1986 en las diferentes localidades de Panamá fue sembrado en enero de 1987 en Río Hato bajo condiciones de riego con el objetivó de aumentar la semilla. De este total en mayo del mismo año se seleccionaron y cosecharon en forma masall.050 líneas; las cuales fueron enviadas al CIAT-Palmira para multiplicación y evaluación. El programa de mejoramiento de arroz del CIAT en septiembre seleccionó sobre 251 líneas un total de 302 plantas individuales que luego entregó al IRTP. Este programa sembró las 302 líneas en octubre de 1987 bajo condiciones de riego-trasplante y en febrero de 1988 seleccionó 46 líneas las cuales fueron incluidas en los viveros de 1988 (VIOAL).
De estas líneas, la CT5747-24-5-2-1-1BA-1BRH2P proveniente del cruce Colombia 1/P 1274-6-8M-1$3 \mathrm{M}-1$ *2/P 2060-F4-2-5-2 se liberó como Fonaiap 2 en Venezuela en el año de 1993. Otra línea de este mismo cruce, la CT5747-38-3-1-1-1A-1BRH-IP, fue nombrada como Porvenir 95 INIA por el programa nacional del Perú en 1995. Cabe destacar que estas variedades son hermanas de la variedad colombiana Oryzica Llanos 5 (CT5747-24-5-4-2), la cual ha mantenido su resistencia a Piricularia por más de seis años en Colombia en siem-bras comerciales.

El programa nacional de Bolivia liberó en 1996 la línea CT6163-8-9-1-2A-IBRH-IP (IR46/IRAT 120/P 1274-6-8M-1-3M-1) como Sacia 5 (Urupé) introducida a través del VIOAL 1988, y que fue evaluada y seleccionada sucesivamente en Alanje (A), Río Hato (RH) y Palmira (P).

En el Cuadro 4 se presenta la genealogía, los progenitores y el año de lanzamiento de las once variedades en siete países desarrolladas con germoplasma seleccionado durante el convenio IDIAPIFCNCIAT. Cinco de estas variedades básicamente para siembras bajo condiciones de riego, como son las liberadas en Nicaragua, Perú, República Dominicana y Venezuela y las restantes para las condiciones de secano favorecido.

La caracterización más reciente de estas variedades a los principales limitantes encontrados bajo condiciones de secano favorecido en la estación CIAT-Santa Rosa, Villavicencio, Colombia, aparece en el Cuadro 5. Todas las variedades, a excepción de Altamira 9 y Juma 64 (ambas del mismo cruce) y Panamá 1537 fueron tolerantes a Piricularia y con excepción de Panamá 1537, Panamá 3621 y Panamá 4721 todos fueron tolerantes a las demás enfermedades fungosas presentes en dicha estación. Se debe resaltar que en Santa Rosa a excepción de Helminthosporiosis, la presión de enfermedades es mayor en comparación con Alanje, Panamá. De nuevo y como se mencionó antes, se corrobora la resistencia a piricularia de las variedades Fonaiap 2 y Porvenir 95, ambas con la misma descendencia de la variedad colombiana Oryzica Llanos 5.

En Palmira y bajo condiciones controladas de campo, todas fueron susceptibles al virus de la hoja blanca; con excepción de Centa AS, todas mostraron tolerancia al daño mecánico del insecto Tagosodes oryzicolus.

En el Cuadro 6 se presentan los datos sobre calidad de grano, molinería y potencial de rendimiento ( $\mathrm{t} / \mathrm{ha}$ ) de estas variedades bajo las condiciones de CIAT-Palmira. Estos datos indican que las variedades presentaron buena calidad de grano y culinaria y un buen potencial 
Cuadro 4. Variedades de arroz liberadas con base en el germoplasma desarrollado por el convenio IDIAP/FCNCIAT.

\begin{tabular}{lllll}
\hline Variedades & Genealogía & Cruzamientos & Año & País \\
\hline Panamá 1537 & P 2062-F4-17-33-1-RH1 & CICA 7// S12-30/P 901-22-11-5-3-2-1B & 1987 & Panamá \\
Panamá 1048 & P 1381-1-8M-2-1B-CH4 & P 931-22/P 918-25//P 914-43/P 918-25 & 1987 & Panamá \\
CENTA A 5 & P 3299-F4-78-3-1B-1 & P 1274-6-8M-1-3M-1//IR1909-1-3-3/IRAT 8 & 1989 & El Salvador \\
JUMA 64 & P 3831-F3-RH38-8-1M-J182 & Oryzica 1//P 1897-15-1-4-1-1B/Costa Rica & 1990 & Rep. Dom. \\
Altamira 9 & P 3831-F3-RH38-8-1M & Oryzica 1//P 1897-15-1-4-1-1B/Costa Rica & 1991 & Nicaragua \\
FONAIAP 1 & P 4070-F3-3-RH3-7-1BA & P 1386-6-8M-1-3M-1//Camponi/Tapuripa & 1993 & Venezuela \\
FONAIAP 2 & CT5747-24-5-2-1-1BA-1BRH-2P & Colombia 1/P1274-6-8M-1-3M-1*2//P 2060-F4-2-5-2 & 1993 & Venezuela \\
Panamá 3621 & P 3621-F2-1-2-8 & Metica 1//Suakoko 8/Ceysvoni & 1993 & Panamá \\
Panamá 4721 & P 4721-F2-10-6 & P 2231-F4-138-6-1B//IR5533-13-1-1/Metica 1 & 1993 & Panamá \\
Porvenir 95 INIA & CT5747-38-3-1-1-1A-1BRH-1P & Colombia 1/P1274-6-8M-1-3M-1*2//P 2060-F4-2-5-2 & 1995 & Perú \\
SACIA- 5 (URUPE) & CT6163-8-9-1-2A-1BRH-1P & IR46/IRAT 120//P 1274-6-8M-1-3M-1 & 1996 & Bolivia \\
\hline
\end{tabular}

Cuadro 5. Caracterización de las variedades liberadas a partir de germoplasma desarrollado durante el convenio IDIAP/FCA/CIAT, en Santa Rosa y Palmira. CIAT. 1996.

\begin{tabular}{|c|c|c|c|c|c|c|c|c|c|c|}
\hline \multirow[b]{2}{*}{ Variedad } & \multicolumn{6}{|c|}{ Santa Rosa ${ }^{1}$} & \multicolumn{4}{|c|}{ Palmira } \\
\hline & FI & $\mathbf{B l}$ & NBI & LSc & BS & GD & Fl & Tag & $\mathbf{H b}$ & País \\
\hline Panamá 1537 & 104 & 6 & 7 & 7 & 1 & 3 & 110 & 1 & 9 & Panamá \\
\hline Panamá 1048 & 104 & 1 & 1 & 5 & 1 & 1 & 108 & 1 & 9 & Panamá \\
\hline CENTA A 5 & 96 & 5 & 1 & 3 & 1 & 1 & 107 & 7 & 9 & El Salvador \\
\hline JUMA 64 & 108 & 8 & 5 & 5 & 5 & 3 & 110 & 1 & 9 & Rep. Dom. \\
\hline Altamira 9 & 102 & 7 & 5 & 3 & 5 & 3 & 112 & 0 & 9 & Nicaragua \\
\hline FONAIAP 1 & 95 & 2 & 1 & 5 & 1 & 1 & 102 & 3 & 7 & Venezuela \\
\hline FONAIAP 2 & 98 & 2 & 1 & 3 & 1 & 1 & 108 & 1 & 7 & Venezuela \\
\hline PANAMA 3621 & 113 & 2 & 1 & 7 & 1 & 3 & 109 & 1 & 9 & Panamá \\
\hline PANAMA 4721 & 108 & 2 & 1 & 7 & 1 & 3 & 110 & 1 & 9 & Panamá \\
\hline PORVENIR 95 INIA & 107 & 3 & 1 & 5 & 1 & 3 & 104 & 3 & 9 & Perú \\
\hline SACIA- 5 (URUPE) & 97 & 2 & 3 & 4 & 3 & 3 & 97 & 5 & 9 & Bolivia \\
\hline
\end{tabular}

de rendimiento el cual fue superior al promedio nacional de arroz obtenido en cada uno de los países en donde se liberaron estas variedades.

Las variedades liberadas han mostrado buena adaptación a condiciones muy diversas del cultivo (riego, secano favorecido y menos favorecido) y buen comportamiento ante variados estreses bióticos y abióticos encontrados en esos ambientes. Esto sugiere que la presión de selección existente en los sitios escogidos para la evaluación y selección de las poblaciones segregantes y la rotación entre sitios fueron factores determinantes para la identificación y liberación de cultivares con una amplia adaptación.
En la mayoría de los casos transcurrió mucho tiempo entre la recepción de una línea promisoria y su liberación como variedad por parte de un programa nacional, máxime cuando se trataba de líneas avanzadas uniformes. Lo cual corrobora lo encontrado por Cuevas-Pérez, et al., 1992, quienes observaron un incremento en el tiempo transcurrido para la liberación de variedades en América Latina, especialmente en el caso de materiales genéticos provenientes de los centros internacionales.

Los beneficios atribuibles a la liberación y adopción del germoplasma mejorado se extendieron mas allá de las fronteras y tiempo contemplados inicialmen- 
Cuadro 6. Calidad del grano y rendimiento ( $\mathrm{t} / \mathrm{ha}$ ) bajo condiciones de riego-siembra directa en Palmira de las variedades liberadas a partir de germoplasma desarrollado durante el Convenio IDIAP/FCAICIAT.

\begin{tabular}{lrrrrrrrl}
\hline \multirow{2}{*}{ Variedad } & \multicolumn{9}{c}{ Calidad de Grano } & & & & \\
\cline { 2 - 5 } & CB & TG & Long. & Amy $(\%)$ & Excelso(\%) & Rend.(t/ha) & País \\
\hline Panamá 1537 & 0.6 & B & L & 30 & 48 & 6.1 & Panamá \\
Panamá 1048 & 004 & B & L & 28 & 50 & 6.1 & Panamá \\
CENTA A 5 & 1.2 & B & L & 24 & 55 & 6.3 & El Salvador \\
JUMA 64 & 0.2 & I & L & 30 & 55 & 10.8 & Rep. Domi. \\
ALTAMIRA 9 & 004 & I & L & 28 & 58 & 7.6 & Nicaragua \\
FONAIAP 1 & 004 & B & EL & 31 & 57 & 8.7 & Venezuela \\
FONAIAP 2 & 1.2 & B & L & 30 & 63 & 8.2 & Venezuela \\
Panamá 3621 & 0.4 & B & L & 29 & 55 & 6.5 & Panamá \\
Panamá 4721 & 004 & I & L & 28 & 51 & 6.0 & Panamá \\
Porvenir 95 INIA & 0.2 & A & L & 24 & 44 & 5.8 & Perú \\
SACIA-5 (URUPE) & 1A & B & EL & 30 & 51 & 6.1 & Bolivia \\
\hline
\end{tabular}

${ }^{1}$ Según escala del Sistema de Evaluación Standar del IRRI.

te en el proyecto colaborativo IDIAP/FCA/CIAT. Este hecho es muy importante ya que según varias fuentes (Lasso, 1992; Sanint, 1992; Martínez, Weber, Sanint, 1988) la mayor parte de la producción de arroz en el área de influencia de este proyecto procede de agricultores pequeños/medianos que cultivan el arroz principalmente bajo condiciones de secano empleando niveles diferentes de tecnificación del cultivo.

La mayoría de las variedades liberadas en base al germoplasma evaluado y seleccionado durante este convenio no han sido aún sembradas lo suficiente en términos de tiempo y área como para evaluar su impacto en la producción local de arroz. No obstante las variedades Panamá 1048 y Centa A 5, de las primeras en ser liberadas, ocupan un porcentaje alto del área sembrada en Panamá y El Salvador y se han diseminado a otras zonas.

Por último, los resultados confirman los beneficios de la cooperación nacional e internacional y demuestran que cuando hay buena fe y voluntad de servicio (McWillian; Dun, 1989), se establecen prioridades y objetivos concretos, se busca la interdependencia de varios grupos y se apropian los recursos necesarios es posible lograr mas de lo que cada uno en particular podría lograr. Por otra parte, los resultados constituyen un indicativo de lo que pueden lograr mecanismos de colaboración/integración similares, como es el caso del Fondo Latinoamericano de Arroz Riego (FLAR) de reciente establecimiento en América Latina y el Caribe.
Los autores agradecen la contribución de la Agencia Internacional para el Desarrollo (AID) con sede en Panamá; el apoyo y entusiasmo que nos brindaron desde el principio, fueron esenciales para la continuidad del proyecto, también agradecen la confianza, sugerencias y apoyo recibido por parte de los directivos del IDIAP, FCA y CIAT. Por último, un reconocimiento especial a las Asociaciones de Productores de Arroz de las provincias de Chiriquí, Coclé y Panamá quienes nos colaboraron y ayudaron en la realización de las pruebas regionales.

\section{LITERATURA CITADA}

CIAT-ANNUAL REPORT RICE PROGRAM. 1986. Rice Annual Report 1985. pp. 160-173

CIAT-ANNUAL REPORT RICE PROGRAM. 1985. Rice Annual Report 1984. pp. 38-40

CIAT-ANNUAL REPORT RICE PROGRAM. 1984. Rice Annual Report 1983. Upland rice breeding: 19-31.

CIAT-ANNUAL REPORT RICE PROGRAM. 1982. Rice Annual Report. 1982. Rice breeding: 1-6

CUEVAS-PÉREZ, F., GUIMARAES, E.P.; MARTÍNEZ, C.P. 1992. Status of rice improvement in Latin America and the Caribbean. In: Rice in Latín America: improvement, management, and marketing. Pro-ceedings VIII Rice Conference for Latin America and the Caribbean. Villahermosa. Tabasco. Mexico. 1991. ed. by Federico Cuevas-Pérez. CIAT. Cali. Colombia. pp. 13-28. 
IDIAP/FCA/CIAT, 1988. Proyecto colaborativo de mejoramiento de arroz. Panamá. Informe de Progreso 1986 y $1987.355 p$.

LASSO-GUEVARA, R 1992. A rice breeding program and its potential contributions to the subsistence farmer. In: Rice in Latin America: improvement, management, and marketing. Proceedings VIII intern. Rice Conference for Latín America and the Caribbean. Villahermosa. Tabasco. Mexico. Nov. 10-16. 1991. Ed. by F. CuevasPérez. CIAT. Cali, Colombia. pp. 43-56.

MARTÍNEZ, C.P.; WEBER, G.; SANINT, L.R 1988. CIAT Rice Program imvolment in Central America: Synopsis. In: Trends in CIAT. Commodities. Internal Document - Economics 1-13. CIAT. Cali, Colombia.
MC WILLlAMS, J.R; DUN, RB. 1989. An act of Faith: Research helps feed the hungry. Australian Center for International Agricultural Research. Camberra. Australia. ISBN 094911943. 40 p.

SANINT, L.R 1996. El arroz de riego en América Latina y el Caribe: Nuevas realidades. Trabajo presentado en curso sobre selección recurrente en arroz. CIAT. 18p.

SANINT, L.R 1992. New rice technologies for Latin America: social benefits, past reminiscences and issues for the future. In: Trends in CIAT commodities 1992. Working document No. 111. CIAT. Cali, Colombia. pp. $1-23$. 